\section{Acesso aos procedimentos de média e alta complexidade no Sistema Único de Saúde: uma questão de judicialização}

\author{
Access to medium and high-complexity procedures \\ in the Brazilian Unified National Health System: \\ a matter of judicialization
}

El acceso a los procedimientos de media y alta complejidad en el Sistema Único de Salud de Brasil: una cuestión de legalización

\begin{abstract}
Lawsuits in healthcare have increased exponentially in Brazil. However, the judicialization of healthcare procedures has not been sufficiently discussed, although such a discussion could broaden the scope of healthcare assessment. This study aimed to analyze the use of court action to ensure access to outpatient and hospital procedures from 1999 to 2009 in the State of Minas Gerais, Brazil. This was a retrospective descriptive study. Procedures were classified according to the Brazilian Unified National Health System (SUS) and the Table on Unified Terminology for Private Healthcare. Coverage by the SUS was 93.5\%. The largest proportions of beneficiaries of such lawsuits lived in the Central and Western regions of the country (26.4\% and $24 \%$, respectively). The most common procedures involved in such cases were clinical admissions, admission to intensive care, and cardiovascular surgery. The study highlights the emerging need for access to medium and high-complexity procedures through extensive rules for coverage.
\end{abstract}

Health Services Accessibility; Health Services Evaluation; Health Services Coverage; Judicial Decisions
Fernanda de Freitas Castro Gomes 1

Mariângela Leal Cherchiglia 1

Carlos Dalton Machado 1

Viviane Cristina dos Santos 1

Francisco de Assis Acurcio 1

Eli Iola Gurgel Andrade 1

\section{Resumo}

Os processos judiciais na área da saúde têm crescido de forma exponencial. A judicialização de procedimentos, no entanto, ainda não foi discutida e pode ampliar o escopo de avaliação da atenção à saúde. O objetivo deste estudo é investigar as ações judiciais para acesso a procedimentos ambulatoriais e hospitalares do Estado de Minas Gerais, Brasil, no período de 1999 a 2009. É um estudo descritivo retrospectivo. Os procedimentos foram classificados pela Tabela Unificada do SUS e pela Tabela de Terminologia Unificada da Saúde Suplementar. Observou-se cobertura pelo SUS de 93,6\%. A residência dos beneficiários localiza-se, principalmente, nas macrorregiões Centro (26,4\%) e Oeste (24\%). Os procedimentos mais solicitados foram internações em leitos comuns, Centro de Terapia Intensiva e cirurgias do aparelho circulatório. Este estudo aponta para as necessidades emergentes de acesso aos procedimentos de média e alta complexidade, mediante uma extensa cobertura normativa.

Acesso aos Serviços de Saúde; Avaliação de Serviços de Saúde; Cobertura de Serviços de Saúde; Decisões Judiciais 


\section{Introdução}

No âmbito internacional, a partir da II Guerra Mundial, o termo judicialização foi cada vez mais associado à ampliação dos direitos humanos fundamentais, resultando em uma notável expansão da via judicial como mecanismo de controle dos demais poderes. Nos marcos do constitucionalismo contemporâneo, o Poder Judiciário ganha legitimidade formal pela Constituição e material ao proteger os direitos fundamentais $1,2,3$. Nesse contexto, a judicialização passa a ser compreendida como a decisão, pelo Poder Judiciário, de questões relevantes do ponto de vista político, social ou moral 4.

No Brasil, a partir da Constituição Federal de 1988, o Estado assume a responsabilidade de garantir a saúde da população, tornando-a um direito fundamental. Pela Constituição, o acesso do cidadão às instâncias de poder também foi ampliado, com a abertura do Poder Judiciário às demandas individuais e coletivas, propiciando um cenário favorável ao chamado "fenômeno da judicialização” 5 . Nos anos noventa, cresce, de forma exponencial, o número de mandados judiciais na saúde ${ }^{6}$. Em 2011, foram contabilizados, pelo Conselho Nacional de Justiça, 240.980 processos na saúde em todo o país 7 , que, em sua maioria, buscavam garantir o acesso das pessoas a medicamentos, procedimentos diagnósticos e terapêuticos.

A fim de compreender esse fenômeno, discussões teóricas têm sido realizadas a partir de concepções denominadas como procedimentalistas e substancialistas, da teoria da reserva do possível e do mínimo existencial 8,9. Nesse sentido, os direitos estabelecidos na Constituição devem ser analisados com neutralidade e democracia, e deve-se adotar o compromisso com os princípios constitucionais. Ao mesmo tempo em que os recursos são limitados, um conjunto de direitos básicos que integram o núcleo da dignidade da pessoa humana deve ser garantido pelo Estado, independente de recursos orçamentários.

Os estudos publicados acerca do fenômeno da judicialização da saúde no Brasil abordam aspectos como a garantia de acesso e cobertura para obtenção de medicamentos 5,10,11, os critérios para deferimento dos pedidos 12,13 e a associação dessas demandas às pressões da indústria farmacêutica e dos atores envolvidos - médicos e advogados 6,14. Outros estudos buscam discutir a caracterização dos processos judiciais nos estados brasileiros, tais como: Santa Catarina 15, São Paulo 16, Rio de Janeiro 17, Minas Gerais 18, Distrito Federal 19 e Mato Grosso 20.

Pelo menos quatro dimensões políticas estão referenciadas na discussão desse fenômeno na literatura consultada: os interesses econômicos associados à pressão para incorporação tecnológica 6,14, a integralidade da assistência terapêutica 21 , o fortalecimento das iniquidades e a sinalização das deficiências e gargalos da assistência à saúde no país 22 .

Desse modo, o crescimento desordenado das ações judiciais nos últimos anos, à medida que impõe gastos inesperados aos orçamentos municipais, estaduais e ao federal, causa grande impacto à gestão do SUS, podendo resultar em agravamento das iniquidades. Ao mesmo tempo, passa a ser inevitável considerar a importância da judicialização como sinalizador das necessidades individuais e coletivas de saúde.

Nesse contexto, ao considerar que a atenção à saúde engloba um conjunto de ações e serviços, percebe-se que a judicialização tem sido relacionada quase exclusivamente à assistência farmacêutica. Nesta pesquisa, não encontramos referências a estudos diretamente relacionados a demandas judiciais por procedimentos ambulatoriais e hospitalares, embora, em geral, façam parte do rol de ações sob recomendação médica que dá origem a um processo judicial em saúde. Entende-se, por procedimento, o detalhamento do método, do processo, da intervenção ou do atendimento do usuário no ambiente e ainda no controle ou acompanhamento das ações complementares ou administrativas 23 .

Neste estudo, o objetivo é investigar os processos judiciais para realização de procedimentos ambulatoriais e hospitalares quanto à cobertura e ao acesso, descrevendo o perfil das ações judiciais ajuizadas contra a Secretaria de Estado de Saúde de Minas Gerais, no período entre 1999 e 2009.

\section{Métodos}

Foi realizado um estudo descritivo retrospectivo cuja unidade de análise constituiu-se de processos judiciais individuais ou coletivos, cadastrados na Secretaria de Estado de Saúde de Minas Gerais, expedidos e recebidos no período de 22 de outubro de 1999 a 20 de outubro de 2009. Os dados foram coletados no período de fevereiro a novembro de 2009, por meio de um formulário previamente testado, o qual estava estratificado em dez categorias: identificação do processo, processo judicial, beneficiários, autor, representante judicial, réu, doença, atendimento, medicamentos e outros pedidos (procedimentos, materiais, equipamentos e outros). O banco de dados assim constituído possui 6.112 processos registrados referentes a medicamentos, materiais, equipamentos, procedimentos e outros. 
A partir desse banco, foram selecionados os processos referentes a procedimentos ambulatoriais e hospitalares nas modalidades: pedidos de transporte para tratamentos, aplicação de medicamentos, consultas, terapias, exames, tratamentos fora do domicílio, internações em leitos comuns ou em Centro de Terapia Intensiva (CTI), cirurgias, transplantes, ações de promoção da saúde e perícias. As variáveis analisadas para descrição do perfil desses processos foram: processo judicial (instância, data, tipo de ação, existência de pedido liminar, resultado do pedido, comarca); beneficiário (sexo, profissão, idade, residência); autor (tipo); representante judicial (tipo); réu (esfera da gestão); doença (diagnóstico, conforme Classificação Internacional de Doenças - CID-10); atendimento (tipo de profissionais, existência de relatório, origem do atendimento) e procedimento (tipo, decisão judicial).

Todos os processos referentes aos procedimentos identificados foram incluídos na análise de perfil das demandas. As respostas nulas ou em branco foram identificadas e expressas nas tabelas como "não informado" para cada variável. Tendo em vista a diversidade de nomes especificados nos processos, para um mesmo procedimento, de modo a possibilitar a análise, os procedimentos foram identificados de acordo com as nomenclaturas correspondentes na Tabela Unificada de Procedimentos, Medicamentos, Órteses, Próteses e Materiais Especiais do Sistema Único de Saúde 23 (Tabela Unificada do SUS), que descreve os procedimentos cobertos pelo SUS. Foi utilizada a competência de julho/2012, a qual descrevia a nomenclatura mais atual do procedimento.

Devido às incompletudes na descrição do procedimento no processo judicial, um procedimento poderia ser identificado com mais de um código ou poderia estar relacionado a um conjunto de procedimentos (por se tratar de solicitações de um tratamento). Nesses casos, os procedimentos identificados na Tabela Unificada do SUS foram os de maior valor monetário. Isso pode ter subestimado o número de procedimentos demandados.

A partir da consulta à Tabela Unificada do SUS, foi elaborado um banco com a classificação por grupo (ações de promoção e prevenção, com finalidade diagnóstica, clínicos, cirúrgicos, transplantes de órgãos, tecidos e células, ações complementares da atenção à saúde), subgrupo (tipo e/ou área de atuação), modalidade de atendimento (ambulatorial; hospitalar; hospital dia; hospitalar, hospital-dia; ambulatorial, hospital-dia; ambulatorial, hospitalar; ambulatorial, atendimento domiciliar; ambulatorial, hospitalar, hospital-dia), complexidade (baixa, média e alta) e cobertura no ano do processo (histórico, código de origem SIA/SIH).

Os procedimentos não identificados na Tabela Unificada do SUS foram alternativamente classificados na Tabela de Terminologia Unificada da Saúde Suplementar para codificação de procedimentos médicos (Tabela TUSS, versão 3.00.00). Os procedimentos não identificados em nenhuma das tabelas foram agrupados nas seguintes categorias: internações, exames, cirurgias, tratamento fora do domicílio, perícias, custeio/reembolso e "sem identificação". Os procedimentos agrupados nessa última classificação e os considerados perícias foram excluídos da análise de cobertura por não conterem informação suficiente para uma avaliação. Foram, entretanto, mantidos nas demais análises.

Os procedimentos judicializados entre os anos de 1999-2007 foram identificados nas Tabelas de Atendimento Ambulatorial (Sistema de Informações Ambulatoriais - SIA) e Atendimento Hospitalar (Sistema de Informações Hospitalares - SIH), nas competências de janeiro e dezembro de cada ano, para verificação de cobertura no ano do processo.

Essa classificação foi validada por três consultores independentes: um médico servidor do Departamento de Controle e Avaliação da Secretaria Municipal de Saúde de Belo Horizonte, um médico colaborador da Secretaria de Estado de Saúde de Minas Gerais e uma enfermeira que atua em uma operadora de planos de saúde.

Foram contempladas informações da entrada do pedido até a autorização para realização do procedimento após deferimento; entretanto, a ausência do desfecho das demandas não permitiu medir o seu impacto.

Neste estudo, foram considerados procedimentos cobertos pelo SUS os que atendiam aos seguintes critérios: possuir código de autorização na Tabela Unificada do SUS ou nas tabelas ambulatoriais (SIA) e hospitalares (SIH); estar classificado como internações, cirurgias, exames e tratamentos fora do domicílio.

Foram considerados sem cobertura pelo SUS, os procedimentos identificados na Tabela TUSS e as solicitações de custeio e reembolso.

Para a avaliação de acesso 24 , foram observados a descrição do perfil das ações e o Índice de Desempenho do SUS (IDSUS) 25, como balizador de acesso aos serviços dos municípios onde foram identificados autores de processos judiciais, ou seja, residentes. O IDSUS foi criado pelo Ministério da Saúde com o objetivo de avaliar o desempenho do SUS quanto ao cumprimento de seus princípios: universalidade do acesso, integralidade da atenção, equidade e de suas diretrizes: regionalização, hierarquização, 
comando único por esfera de gestão e responsabilidade tripartite. Para efeito dessa análise, foi feita correlação entre o número de procedimentos demandados judicialmente entre 1999-2009 e as notas de desempenho geral dos municípios em 2011. Foram também analisadas as médias de desempenho por macrorregião de quatro indicadores de "acesso obtido" (2007-2009) 25 que avaliam o acesso à Atenção Ambulatorial e Hospitalar de média e alta complexidade: razão de procedimentos ambulatoriais de alta complexidade; razão de procedimentos clínicos de média complexidade; razão de procedimentos clínicos de alta complexidade e razão de procedimentos cirúrgicos de alta complexidade.

São limitações do estudo as diferenças temporais na análise dos processos (1999-2009) e na base de dados do IDSUS (2007-2009). Apesar de a base do IDSUS não contemplar o mesmo período de análise dos processos judiciais, considera-se viável a sua utilização como balizador de desempenho, tendo, em vista, a ausência desses dados para períodos anteriores. Além disso, não há registros de grandes alterações estruturais na ampliação do acesso aos serviços nas regiões analisadas e o período em análise no estudo.

Os resultados descritivos foram obtidos utilizando frequências e porcentagens para as características das diversas variáveis categóricas e da obtenção de média e desvio-padrão para as variáveis contínuas. Foi verificada correlação entre o número de processos por população dos municípios. Considerou-se um nível de 5\% de significância. A análise foi realizada no software PASW Statistics, versão 18.0 (IBM Corp., Armonk, Estados Unidos).

Este trabalho é resultado do projeto de pesquisa intitulado Avaliação de Cobertura, Acesso e Qualidade da Assistência Farmacêutica, Garantidos pelas Decisões Judiciais em Minas Gerais, desenvolvido pelo Grupo de Pesquisa em Economia da Saúde da Faculdade de Medicina, da Universidade Federal de Minas Gerais (CNPq/GPES/ FM/UFMG), com aprovação pelo Comitê de Ética em Pesquisa (COEP) da UFMG, emitido em 24 de setembro de 2008 (parecer no ETIC 292/08).

\section{Resultados}

Entre os 6.112 processos judiciais registrados contra o Estado de Minas Gerais, 783 (12,8\%) se referem a 1.002 procedimentos para atender a 908 usuários. A maior demanda ocorreu no triênio de 2007-2009 (80,8\%), e 76\% dos processos relacionados aos procedimentos foram deferidos.

As demandas judiciais abrangem 122 cidades, representando 14\% do Estado. Há correlação entre o número de processos e a população $(r$ = $0,520 ; \mathrm{p}<0,001)$. Nas comarcas de Belo Horizonte $(22,3 \%)$ e Divinópolis (19,8\%), pertencentes às macrorregiões Centro e Oeste, respectivamente, foram ajuizados a maior parte dos processos. Os beneficiários eram residentes no mesmo município da comarca em $81 \%$ dos casos. Em municípios pequenos, tais como Carmo do Cajuru e Ritanópolis, as razões entre o número de processos e o tamanho da população foram mais altas do que nos demais municípios.

Houve maior frequência de ações individuais judicializadas em la instância (95,5\%) e de pedidos de liminares (98\%). A maioria das liminares foi deferida $(71 \%)$. Em $78,8 \%$ das solicitações, houve autorização para realização do procedimento por parte da Secretaria de Estado de Saúde de Minas Gerais. Há maior frequência de beneficiários como autores das ações. O Ministério Público, porém, foi autor em mais de um terço delas. As representações judiciais públicas ou gratuitas, somadas às sem representação totalizam 50\%. O Estado figurou como réu em 100\% das ações. Em $63 \%$ dos casos, os municípios também figuraram como réu (Tabela 1).

Dentre as informações disponíveis, o perfil dos beneficiários se caracteriza pelo predomínio de homens $(54,1 \%)$, idade acima de 50 anos, aposentados e donas de casa. Dentre as organizações de atendimento do paciente, as mais frequentes foram as entidades beneficentes sem fins lucrativos e as unidades de saúde municipais e estaduais (Tabela 2).

Os 1.002 procedimentos demandados judicialmente foram descritos em 4 categorias: identificados na Tabela Unificada do SUS (633); identificados na Tabela TUSS (28) e não identificados na Tabela TUSS (15). Os procedimentos não identificados em nenhuma dessas tabelas foram denominados “incompletos" (327). Nesse grupo, foram reconhecidos: solicitações de internações, cirurgias, exames, tratamento fora do domicílio (278), custeio e reembolso (17), perícias (4) e "sem identificação" (28).

Conforme as descrições na Tabela Unificada do SUS, predominaram os grupos de procedimentos cirúrgicos $(37,2 \%)$ e com finalidade diagnóstica (26,6\%). Dentre os subgrupos, destacou-se o de ações relacionadas ao atendimento $(14,2 \%)$, sendo a internação em CTI $(14,2 \%)$ o procedimento mais judicializado. Também merecem destaque o subgrupo aparelho circulatório (11,2\%), consultas/atendimentos (9,3\%) e aparelho de visão (7\%) (Tabela 3).

Os procedimentos mais solicitados na Tabela TUSS foram PET Scan (25,8\%), Angioplastia com Stent Farmacológico (22,6\%) e Oxigenoterapia Hiperbárica $(16,1 \%)$ (Tabela 4). Como a versão 
Perfil dos processos judiciais solicitando procedimentos ambulatoriais e hospitalares ajuizados contra o Estado de Minas Gerais, Brasil, 1999-2009.

\begin{tabular}{|c|c|c|}
\hline Variável & $\mathbf{n}$ & $\%$ \\
\hline \multicolumn{3}{|l|}{ Instância da petição inicial ( $\mathrm{n}=783$ ) } \\
\hline Fórum & 571 & 72,9 \\
\hline Tribunal & 35 & 4,5 \\
\hline Federal & 79 & 10,1 \\
\hline Federal especial & 95 & 12,1 \\
\hline Federal itinerante & 3 & 0,4 \\
\hline \multicolumn{3}{|l|}{ Tipo de ação proposta $(n=783)$} \\
\hline Ação civil pública & 107 & 13,7 \\
\hline Ação ordinária & 573 & 73,2 \\
\hline Mandado de segurança & 69 & 8,8 \\
\hline Outros & 34 & 4,3 \\
\hline Pedido de liminar $(n=783)$ & 771 & 98,5 \\
\hline Houve deferimento $(n=771)$ & 654 & 84,8 \\
\hline Houve deferimento parcial $(n=771)$ & 50 & 6,5 \\
\hline Houve suspensão $(n=771)$ & 19 & 2,5 \\
\hline Indeferimento $(n=771)$ & 48 & 6,2 \\
\hline \multicolumn{3}{|l|}{ Decisão Judicial ( $n=1.002)$} \\
\hline Ainda não há decisão & 45 & 4,7 \\
\hline Entrega do pedido & 760 & 78,8 \\
\hline Indeferimento (liminar ou sentença) & 134 & 13,9 \\
\hline Processo extinto (Art. 267) & 5 & 0,5 \\
\hline Suspensão da entrega do pedido (liminar) & 21 & 2,2 \\
\hline Não se aplica & 12 & - \\
\hline Não informado & 25 & - \\
\hline \multicolumn{3}{|l|}{ Autor $(n=785)$} \\
\hline Beneficiários & 483 & 61,5 \\
\hline Ministério Público Estadual & 242 & 30,8 \\
\hline Ministério Público Federal & 58 & 7,4 \\
\hline Defensoria Pública & 1 & 0,1 \\
\hline Município & 1 & 0,1 \\
\hline \multicolumn{3}{|l|}{ Tipo de representante judicial do autor $(n=851)$} \\
\hline Advogado & 267 & 49,1 \\
\hline Defensor Público & 180 & 33,1 \\
\hline Núcleo de assistência jurídica & 10 & 1,8 \\
\hline Sem representação & 87 & 16,0 \\
\hline Não informado & 307 & - \\
\hline \multicolumn{3}{|l|}{ Réu da petição ( $n=793$ ) } \\
\hline União & 179 & 22,9 \\
\hline Estado & 783 & 100,0 \\
\hline Município & 495 & 63,2 \\
\hline Hospitais & 30 & 3,8 \\
\hline
\end{tabular}

consultada dessa tabela foi a do ano de 2012, não é possível relacionar a incorporação desses procedimentos ao ano de entrada dos processos. Ainda em relação à Tabela 4 , vale ressalvar que, embora os procedimentos não tenham sido identificados na Tabela Unificada do SUS, a partir da nomenclatura registrada no processo, não deve ser descartada a possibilidade de estarem contidos na tabela com outra nomenclatura. 
Tabela 2

Perfil dos beneficiários dos procedimentos judiciais solicitando procedimentos ambulatoriais e hospitalares ajuizados contra o Estado de Minas Gerais, Brasil, 1999-2009.

\begin{tabular}{|c|c|c|}
\hline Variável & n & $\%$ \\
\hline \multicolumn{3}{|l|}{$\operatorname{Sexo}(n=908)$ * } \\
\hline Feminino & 411 & 45,9 \\
\hline Masculino & 484 & 54,1 \\
\hline Não informado & 14 & - \\
\hline \multicolumn{3}{|l|}{ Faixa etária [anos] $(n=908)$ * } \\
\hline $1-9$ & 41 & 9,1 \\
\hline $10-19$ & 42 & 9,3 \\
\hline $20-29$ & 23 & 5,1 \\
\hline $30-39$ & 33 & 7,3 \\
\hline $40-49$ & 47 & 10,4 \\
\hline $50-59$ & 57 & 12,7 \\
\hline 60 ou mais & 207 & 46,1 \\
\hline Não informado & 530 & - \\
\hline \multicolumn{3}{|l|}{ Ocupação $(n=908)$ * } \\
\hline Forças Armadas, policiais e bombeiros militares & 2 & 0,6 \\
\hline \multicolumn{2}{|l|}{ e gerentes } & 2,2 \\
\hline Profissionais das ciências e das artes & 10 & 3,1 \\
\hline Técnicos de nível médio & 15 & 4,6 \\
\hline Trabalhadores de serviços administrativos & 3 & 0,9 \\
\hline Trabalhadores dos serviços, vendedores do comércio em lojas e mercados & 52 & 16,1 \\
\hline Trabalhadores agropecuários, florestais, da caça e da pesca & 6 & 1,9 \\
\hline Trabalhadores da produção de bens e serviços industriais & 30 & 9,3 \\
\hline Trabalhadores de manutenção e reparação & 2 & 0,6 \\
\hline Aposentado & 102 & 31,5 \\
\hline Estudante & 18 & 5,6 \\
\hline Dona de casa & 55 & 17,0 \\
\hline Sem profissão & 1 & 0,3 \\
\hline Desempregado & 20 & 6,2 \\
\hline Não informado & 585 & - \\
\hline \multicolumn{3}{|l|}{ Natureza da organização } \\
\hline Administração direta da saúde, Ministério da Saúde, Secretaria Estadual, Secretaria Municipal & 35 & 20,1 \\
\hline Administração direta de outros órgãos, Ministério da Educação, Marinha, outros & 15 & 8,6 \\
\hline Administração indireta - autarquias & 29 & 16,7 \\
\hline Administração indireta - fundação pública & 4 & 2,3 \\
\hline Cooperativa & 1 & 0,6 \\
\hline Empresa privada & 29 & 16,7 \\
\hline Entidade beneficente sem fins lucrativos & 56 & 32,2 \\
\hline Fundação privada & 2 & 1,1 \\
\hline Serviço social autônomo & 3 & 1,7 \\
\hline Não se aplica & 162 & - \\
\hline Não informado & 641 & - \\
\hline
\end{tabular}

* $3 \%$ dos processos tiveram mais de um autor. 
Descrição dos procedimentos ambulatoriais e hospitalares dos processos judiciais ajuizados contra o Estado de Minas Gerais, Brasil, conforme classificação na Tabela Unificada do SUS, 1999-2009 ( N = 633).

\begin{tabular}{|c|c|c|}
\hline Grupo/Subgrupo & $\mathrm{n}$ & $\%$ \\
\hline \multicolumn{3}{|l|}{ Ações de promoção e prevenção à saúde $(n=1 ; 0,2 \%)$} \\
\hline Ações coletivas/individuais em saúde & 1 & 0,2 \\
\hline \multicolumn{3}{|l|}{ Procedimentos com finalidade diagnóstica ( $n=168 ; 26,6 \%$ ) } \\
\hline Ultrassonografia, endoscopia, radiologia interventiva & 39 & 6,2 \\
\hline Diagnósticos em especialidades & 39 & 6,2 \\
\hline Ressonância magnética & 35 & 5,5 \\
\hline Coleta de material, laboratorial, clínico, anatomia patológica, teste rápido & 33 & 5,2 \\
\hline Medicina nuclear em vivo, tomografia & 22 & 3,5 \\
\hline \multicolumn{3}{|l|}{ Procedimentos clínicos ( $n=107 ; 16,8 \%)$} \\
\hline Consultas/Atendimentos & 59 & 9,3 \\
\hline Tratamentos clínicos (outras especialidades) & 34 & 5,4 \\
\hline Tratamento em oncologia & 4 & 0,6 \\
\hline Terapias especializadas e fisioterapia & 10 & 1,6 \\
\hline \multicolumn{3}{|l|}{ Procedimentos cirúrgicos ( $n=235 ; 37,2 \%$ ) } \\
\hline Aparelho circulatório & 71 & 11,2 \\
\hline Aparelho de visão & 44 & 7,0 \\
\hline Sistema osteomuscular & 40 & 6,3 \\
\hline Aparelho digestivo, órgãos anexos e parede abdominal & 36 & 5,7 \\
\hline Outras & 44 & 7,0 \\
\hline Transplantes de órgãos, tecidos e células ( $n=2 ; 0,3 \%)$ & & 0,0 \\
\hline Transplantes de órgãos, tecidos e células & 2 & 0,3 \\
\hline \multicolumn{3}{|l|}{ Ações complementares da atenção à saúde ( $n=120 ; 19 \%)$} \\
\hline Ações relacionadas ao atendimento * & 90 & 14,2 \\
\hline Autorização/Regulação & 30 & 4,7 \\
\hline Total & 633 & 100,0 \\
\hline
\end{tabular}

* Todos os procedimentos classificados nesse grupo referem-se a internações em CTI.

No resultado da avaliação de cobertura pelo SUS, 630 (64,9\%) procedimentos identificados na Tabela Unificada do SUS possuíam cobertura no ano do processo; três procedimentos não constavam nas tabelas dos anos de origem do processo; 31 estavam no rol de cobertura da Agência Nacional de Saúde Suplementar (ANS); e 11 não possuíam cobertura pelo SUS nem pelos planos de saúde. Os 278 procedimentos de internações, cirurgias, exames e tratamento fora do domicílio foram reconhecidos na cobertura SUS e representaram $28,7 \%$ do total de procedimentos avaliados. Dos 970 (96,8\%) procedimentos avaliados, 93,6\% tinham cobertura pelo SUS, e não foi possível avaliar a cobertura de $32(3,2 \%)$ procedimentos (Tabela 5).

O procedimento mais solicitado foi internação (135), classificado no grupo de cirurgias, internações, exames e tratamento fora do domicílio. O segundo pedido mais frequente foi ressonância magnética (35).
Na classificação dos procedimentos por modalidade, foram agrupadas todas as designações contendo "hospitalar", totalizando 77\%. Predominaram os procedimentos de média complexidade $(51,1 \%)$ e os de alta complexidade $(44,4 \%)$. Observou-se que $80,8 \%$ dos procedimentos clínicos eram de média complexidade, e 53,6\% dos procedimentos cirúrgicos eram de alta complexidade (53,6\%). Apesar de não ter sido utilizada nenhuma referência para identificação da complexidade dos demais procedimentos, percebe-se que houve predomínio de solicitações de internação, internação em CTI, procedimentos cirúrgicos e exames com tecnologia avançada, que poderiam ser denominados como de média ou alta complexidade.

De acordo com a CID-10, $684(68,2 \%)$ procedimentos continham diagnósticos. Observou-se que as doenças mais frequentes eram do aparelho circulatório (26,5\%); sistema osteomuscular e tecido conjuntivo (15\%); endócrinas, nutricio- 
Descrição dos procedimentos ambulatoriais e hospitalares ajuizados contra o Estado de Minas Gerais, Brasil, 1999-2009, conforme classificação pela Tabela de Terminologia Unificada de Saúde Suplementar (Tabela TUSS) $(n=42)$.

\begin{tabular}{|c|c|c|}
\hline Descrição & $\mathbf{n}$ & $\%$ \\
\hline \multicolumn{3}{|l|}{ Tabela TUSS ( $n=31 ; 73 \%)$} \\
\hline PET Scan & 8 & 36,4 \\
\hline Angioplastia com stent farmacológico & 7 & 31,8 \\
\hline Oxigenoterapia hiperbárica & 5 & 22,7 \\
\hline Plasmaférese terapêutica & 2 & 9,1 \\
\hline Cirurgia corretiva de miopia e astigmatismo & 2 & 9,1 \\
\hline Implante de anel de ferrara & 2 & 9,1 \\
\hline Angiofluoresceinografia - monocular & 1 & 4,5 \\
\hline Ablação por radiofrequência & 1 & 4,5 \\
\hline Manometria retal & 1 & 4,5 \\
\hline Implante de esfíncter artificial & 1 & 4,5 \\
\hline Tomografia de coerência óptica & 1 & 4,5 \\
\hline \multicolumn{3}{|l|}{ Não informado na Tabela TUSS ( $\mathrm{n}=11 ; 26 \%$ ) } \\
\hline Implantação de bomba de infusão contínua de insulina & 4 & 36,4 \\
\hline Técnicas de fertilização & 2 & 18,2 \\
\hline Tomografia computadorizada helicoidal & 1 & 9,1 \\
\hline Cintilografia com octreoscan & 1 & 9,1 \\
\hline Ressonância magnética tridimensional & 1 & 9,1 \\
\hline Transplante de uretra & 1 & 9,1 \\
\hline Tratamento de reeducação postural global & 1 & 9,1 \\
\hline
\end{tabular}

Tabela 5

Cobertura pelo SUS dos procedimentos ambulatoriais e hospitalares judicializados em Minas Gerais, Brasil, $1999-2009$ $(\mathrm{N}=1.002)$.

\begin{tabular}{|c|c|c|c|c|}
\hline \multirow[t]{2}{*}{ Categorias } & \multicolumn{2}{|c|}{ Sim } & \multicolumn{2}{|c|}{ Não } \\
\hline & $\mathbf{n}$ & $\%$ & $\mathbf{n}$ & $\%$ \\
\hline Tabela Unificada do SUS & 630 & 64,9 & 3 & 0,3 \\
\hline Tabela TUSS & 0 & 0,0 & 31 & 3,2 \\
\hline Não encontrado na Tabela TUSS & 0 & 0,0 & 11 & 1,1 \\
\hline \multicolumn{5}{|l|}{ Incompletos } \\
\hline Internações, cirurgias, exames, tratamento fora do domicílio & 278 & 28,7 & 0 & 0,0 \\
\hline Custeio e reembolso & 0 & 0,0 & 17 & 1,7 \\
\hline
\end{tabular}

Tabela TUSS: Tabela de Terminologia Unificada de Saúde Suplementar.

Nota: sem condições de avaliar cobertura: 4 perícias e 28 procedimentos sem identificação.

nais e metabólicas (7,9\%); olhos e anexos (7,4\%); e neoplasias $(7,2 \%)$.

Ao analisar o IDSUS naqueles municípios em que houve processos, observou-se que a macrorregião Nordeste, onde foram registrados 37 pedidos de procedimentos, alcançou a menor média de desempenho em relação às demais macrorre- giões $(5,2 ; \mathrm{DP} \pm 0,58)$. A melhor média de desempenho foi da macrorregião Jequitinhonha $(6,7$; $\mathrm{DP} \pm 0,03$ ), onde foram registrados dois pedidos de procedimentos. Na macrorregião Centro, que originou a maior demanda por procedimentos (264), os municípios tiveram desempenho médio de 6,0 (DP $\pm 0,58$ ). A macrorregião Oeste, também 
com demanda elevada por procedimentos, obteve média de desempenho de 5,8 ( $\mathrm{DP} \pm 1,9)$.

Em relação ao indicador razão média de internações clínico-cirúrgicas de alta complexidade e população residente, os desempenhos das macrorregiões Nordeste, Jequitinhonha, Centro e Oeste foram de 0,6 (DP \pm 0$) ; 3,8(\mathrm{DP} \pm 0) ; 4,3$ $(\mathrm{DP} \pm 0,64)$ e $4,5(\mathrm{DP} \pm 1,68)$, respectivamente Quanto ao indicador razão média de procedimentos ambulatoriais de média complexidade e população residente, os desempenhos dessas macrorregiões foram de 0,31 (DP \pm 0$) ; 3,8$ $(\mathrm{DP} \pm 0) ; 4,3(\mathrm{DP} \pm 1,30)$ e $4,5(\mathrm{DP} \pm 0,27)$. Nas macrorregiões Nordeste e Jequitinhonha, não houve demanda judicial para internações clínico-cirúrgicas de média complexidade. As macrorregiões Centro e Oeste obtiveram as médias $5,5(\mathrm{DP} \pm 0,29)$ e $2,8(\mathrm{DP} \pm 0)$ para a razão média de internações clínico-cirúrgicas de média complexidade e população residente. Dentre essas quatro macrorregiões, somente a Centro e Oeste tiveram demandas judiciais para procedimentos ambulatoriais de alta complexidade, 4,8 $(\mathrm{DP} \pm 1,32)$ e $3,1(\mathrm{DP} \pm 3,1)$, respectivamente.

Ao analisar as médias de desempenhos de todas as macrorregiões desses quatro indicadores, observa-se que as médias variam de 2,45 a 5,28, sendo a menor média, a de internações clínicocirúrgicas de alta complexidade e população residente, também com a maior demanda de procedimentos. Entretanto, não houve correlação entre o número de procedimentos e as notas desses indicadores por município $(r=0,431 ; p>$ $0,05)$.

\section{Discussão}

A judicialização de procedimentos ambulatoriais e hospitalares se caracteriza por um perfil diferenciado da judicialização de medicamentos, com multiplicidade de médicos e advogados, em contraposição à estreita vinculação entre escritórios de advocacia e a profissionais médicos nas ações relacionadas a medicamentos, conforme sinalizaram os estudos de Campos Neto 14, Pereira et al. 15, Marques \& Dallari 16 e Chieffi \& Barata 11. No caso da judicialização de procedimentos, destacaram-se as representações judiciais públicas ou gratuitas, com importante atuação do Ministério Público.

A idade acima de 50 anos, sexo masculino, com ocupação nas categorias aposentados e donas de casa foram mais frequentes. Os procedimentos mais solicitados foram: internação, internação em CTI, ressonância magnética, consulta em atenção especializada, gastroplastia, implante de marca-passo de câmara dupla, tra- tamento em psiquiatria, artroplastia de quadril e joelho e injeção intravítrea, respectivamente.

Quanto ao perfil de morbidade, as doenças mais frequentes relacionadas nos processos corroboram a transição epidemiológica e o estudo de carga de doenças de Minas Gerais 21,22. Os diagnósticos se assemelharam em relação a doenças cardiovasculares e diabetes como encontrados em Campos Neto et al. 14 e Marques \& Dallari 16 .

Conforme discutido por Schramm et al. 26 , o envelhecimento da população traz uma mudança no perfil epidemiológico que aumenta a demanda por serviços de saúde. Para tanto, torna-se imprescindível fortalecer as estratégias de utilização dos recursos.

Por outro lado, algumas características se assemelham à judicialização de medicamentos. Observa-se, por exemplo, concentração de processos nas capitais e em grandes municípios. Esses dados coincidem com achados de Borges \& Ugá 13 e Bellato et al. 20. De maneira similar a Ventura et al. 5 e Marques \& Dallari 16, constatouse pelo predomínio de pedidos de liminar e deferimentos.

Um importante achado neste estudo referese ao alto percentual de cobertura de procedimentos do SUS para os procedimentos judicializados. Observando os resultados (Tabela 5), além de a maioria dos procedimentos classificados $(64,9 \%)$ serem cobertos pelo SUS, quando a eles se somam os classificados como internações, cirurgias, exames e tratamento fora do domicílio $(28,7 \%)$, atinge-se uma relevante cobertura de 93,6\%. Não foi possível precisar se a cobertura dos procedimentos descritos como internações, cirurgias e exames foi total, mas considerou-se que o principal objeto do processo não seria a realização do procedimento, e, sim, a garantia de acesso ao atendimento, dificultado pela ausência de vagas e outros fatores administrativos. $\mathrm{O}$ fato a destacar, nesse caso, é que a judicialização passa a "funcionar" como via de acesso a ações que são componentes do rol de oferta do SUS.

Machado et al. 18 apontam o fato da ocorrência da judicialização de medicamentos mesmo quando constantes em listas oficiais do SUS. Nesse caso, os autores chamam atenção para a vinculação entre os protocolos clínicos de diretrizes terapêuticas e determinadas entidades mórbidas. A superação de tais incongruências parece estar na base de algumas ações recentes que têm incidência na ampliação de cobertura do SUS. A primeira delas ocorreu em 2008, com a criação da Tabela Unificada do SUS, cuja ampliação do rol de procedimentos é constante. Mais recentemente, a publicação do Decreto no $7508 / 201127$, que estabeleceu a atualização da 
Relação Nacional de Medicamentos (RENAME), a criação da Relação Nacional de Ações e Serviços de Saúde (RENASES), o processo de formação das Regiões de Saúde e o Contrato Organizativo da Ação Pública da Saúde (COAP). Outra ação foi a criação da Comissão Nacional de Incorporação de Tecnologias (CONITEC) por meio do Decreto no 7646/2011 28.

Conforme dados disponíveis, não foi possível identificar as razões que levaram os usuários a reivindicarem acesso à assistência à saúde por via judicial. Mas, tendo em vista o elevado percentual de procedimentos contemplados na Tabela Unificada do SUS (tomado como indicador de cobertura), os fatores tempo de diagnóstico e ausência de vagas podem ser fortes indícios de dificuldades de acesso. Considerando, por exemplo, que o procedimento mais demandado foi internação, inclusive internação em CTI, pode-se discutir que: conforme parâmetros de cobertura assistencial 29, para cada mil habitantes, devem ser disponibilizados 2,5 a 3 leitos hospitalares e 4 a 10\% de leitos de CTI do total de leitos hospitalares. Entretanto, em Minas Gerais, estão registrados atualmente 24.936 leitos hospitalares, cerca de $50 \%$ a menos do recomendado, considerando a população de Minas Gerais com 19.597.330 habitantes ${ }^{30}$. Os leitos de UTI somam 2.794 leitos (Cadastro Nacional de Estabelecimentos de Saúde. http://www.cnes.data sus.gov.br, acessado em 10/Fev/2013), em torno de $11 \%$ do total de leitos hospitalares existentes, mas correspondendo a apenas 6\% (dos 48.993) dos leitos hospitalares recomendados. É importante considerar as diferenças regionais em que há maior número de leitos nos municípios com maior resolubilidade para atendimento. Independente da redução de leitos hospitalares no Brasil 30, é importante destacar, conforme afirmam Campos \& Amaral ${ }^{31}$, que as respostas à pressão por incorporação de procedimentos de alto custo têm sido mais rápidas do que as ações para a desospitalização. Dessa forma, esses dados podem ser indicadores da necessidade de reprogramação do número de leitos, de intensificação no gerenciamento dos existentes e, principalmente, do desenvolvimento de políticas que incentivem a desospitalização e assegurem a eficácia da atenção básica.

Assim, as barreiras de acesso ao atendimento no SUS não podem ser negligenciadas. De acordo com o estudo de Carvalho \& Gianini 32, que discutiram as iniquidades de tempo de espera do usuário do SUS, a diferença chega a 5,5 meses ( $\mathrm{p}<0,001)$ em relação ao atendimento privado. Em pesquisa de opinião do Instituto de Pesquisa Econômica Aplicada (IPEA) 33 com os usuários do SUS, essas dificuldades também foram men- cionadas. Entre as conclusões, há indicações para o aumento do número de médicos e redução do tempo de espera entre a marcação e a realização da consulta, bem como a diminuição do tempo para atendimento nas urgências. Portanto, o atendimento em tempo oportuno pode ser considerado uma emergente necessidade de saúde no cenário atual do sistema de saúde.

A não correlação entre o número de procedimentos por município e a análise das médias de desempenho obtidas em cada indicador que compõe o IDSUS 25 aponta uma discrepância entre a judicialização e a avaliação de desempenho desses municípios, ou seja, a via judicial tem sido utilizada mesmo em municípios com melhores médias de desempenho, assim como há baixa demanda judicial em municípios com desempenhos ruins. De um modo geral, isso pode indicar a necessidade de ações que resultem em melhoria do acesso aos serviços de saúde de Minas Gerais.

Dentre os casos não cobertos pelo SUS, estão os procedimentos Oxigenoterapia Hiperbárica e PET Scan. Destaca-se que tais procedimentos só foram incorporados pela ANS a partir de janeiro de 2012. Isso pode estar relacionado ao maior grau de vulnerabilidade desse seguimento para a incorporação de novas tecnologias.

O presente estudo tem, como limitação, as incompletudes inerentes à fonte pesquisada (expedientes administrativos e documentais dos processos judiciais) que levaram a perdas nas variáveis - idade, ocupação e diagnóstico da doença. Também vale notar que, ao deferir um tratamento, nem sempre é detalhado (pelo juiz/médico) o conjunto de procedimentos que o constitui. Dessa forma, é preciso considerar que o volume total de procedimentos pode estar subestimado.

Outro aspecto refere-se à necessidade de ampliar o escopo de análise do fenômeno, desde aspectos descritivos, para análises qualitativas de maior alcance temporal e de desfechos.

Por outro lado, na medida em que não foi verificada a procedência e efetiva realização dos procedimentos, o número de processos efetivamente atendidos pode ter sido menor.

Os resultados obtidos por este estudo sinalizam gargalos nas linhas de cuidado e dificuldades na garantia da integralidade da atenção à saúde pelo SUS. Ou seja, nos dispositivos legais, o sistema oferta o serviço de forma universal, mas não consegue atender as demandas integralmente. Além disso, essas ações judiciais indicam uma dificuldade do SUS para suprir as diversas necessidades de utilização dos serviços, discutidas por Campos ${ }^{34}$, em atendimento ao direito à vida. $\mathrm{O}$ aumento das demandas por procedimentos de média e alta complexidades e, consequente- 
mente, a dificuldade de acesso são justificadas por Spedo et al. 35 devido à reprodução do modelo hegemônico, baseado em procedimentos e em uma baixa resolutividade da atenção básica. Outra justificativa é percebida por Fleury 22 ao discutir o modelo de Estado comprador de serviços, não mais provedor ou gestor, e a introdução da lógica de mercado na gestão dos serviços, que tende a distorcer o direito à saúde. Nesse cenário, vale salientar que o pagamento por procedimento, baseado em séries históricas, pode não representar as necessidades reais de saúde.

\section{Considerações finais}

Este estudo retoma as questões de acesso e cobertura relacionadas no fenômeno de judicialização da saúde no Brasil. Chama a atenção para as necessidades de saúde da população, garantidas constitucionalmente a partir do princípio de integralidade. Necessidades essas que não encontram a mesma prioridade que o tratamento medicamentoso no fenômeno da judicialização.
Em um primeiro momento, o Poder Judiciário foi criticado por intervir na área da saúde, desconsiderando as normas e políticas de gestão estabelecidas e fazendo cumprir a lei, baseandose no direito à vida. Em seguida, foi questionada a interpretação dos princípios de integralidade, equidade e universalidade ao mesmo tempo em que surge a desconfiança de parcerias entre a indústria farmacêutica, médicos e advogados no processo de judicialização. É debatida, então, a capacidade técnica do Poder Judiciário para intervir na organização e gestão do sistema de saúde.

Nesse momento, vem à tona uma nova percepção, conforme destaca Fleury 22, de que a judicialização possa ser aliada do SUS, por sinalizar as deficiências e estimular a reflexão para novas políticas, de forma a reduzir a distância entre o SUS estabelecido no arcabouço normativo e o SUS que executa as ações e serviços de saúde. Este estudo reforça essa perspectiva ao levantar as necessidades emergentes de acesso aos procedimentos de média e alta complexidade, mediante uma extensa cobertura normativa.

\section{Resumen}

Las demandas en materia de salud han crecido de manera exponencial. La judicialización de los procedimientos, sin embargo, aún no se ha discutido y podría ampliar el alcance de la evaluación de la atención sanitaria. El objetivo de este estudio es investigar los procedimientos judiciales para el acceso a la atención ambulatoria y hospitalaria del Estado de Minas Gerais, Brasil, durante el periodo 1999-2009. Se trata de un estudio descriptivo retrospectivo. Los procedimientos fueron clasificados por la Tabla SUS Unificada y la Mesa de Terminología Unificada de Seguros de Salud. Se observó una cobertura de un 93,6\% en el SUS. La estancia de los beneficiarios estaba ubicada principalmente en el macro-centro $(26,4 \%)$ y zona occidental (24\%). Los procedimientos más solicitados son las admisiones para las camas de hospital, en la unidad de cuidados intensivos y cirugía del aparato circulatorio. Este estudio apunta a las nuevas necesidades de acceso, a los procedimientos de media y alta complejidad, a través de amplias normas de cobertura.

Accesibilidad a los Servicios de Salud; Evaluación de Servicios de Salud; Cobertura de los Servicios de Salud; Decisions Judiciales 


\section{Colaboradores}

Todos os autores contribuíram na concepção, planejamento, desenho, análise e interpretação dos dados, revisão crítica do conteúdo e aprovação da versão final do manuscrito.

\section{Agradecimentos}

Os autores agradecem à Secretaria Municipal de Saúde de Belo Horizonte, em especial, ao colaborador Oswaldo Geraldo da Silva Teixeira, do Departamento de Controle e Avaliação, pela validação dos dados; à Amanda Cristina de Souza Andrade pela colaboração na análise estatística dos dados; ao Grupo de Pesquisa em Economia da Saúde da Universidade Federal de Minas Gerais; ao CNPq e à FAPEMIG.

\section{Referências}

1. Vallinder T, Tate CN. The global expansion of judicial power: the judicialization of politics. New York: New York University; 1995.

2. Carvalho ER. Em busca da judicialização da política no Brasil: apontamentos para uma nova abordagem. Revista de Sociologia e Política 2004; 23:115-26.

3. Benedetti ARM. Judicialização da política: a construção de um conceito constitucional nos cenários de expansão do poder judiciário [Dissertação de Mestrado]. Curitiba: Pontifícia Universidade Católica do Paraná; 2011.

4. Barroso LR. Constituição, democracia e supremacia judicial: direito e política no Brasil contemporâneo. Revista Jurídica da Presidência da República 2010; 12:4-41.

5. Ventura M, Simas L, Pepe VLE, Schramm FL. Judicialização da saúde, acesso à justiça e a efetividade do direito à saúde. Physis (Rio J.) 2010; 20:7-10.

6. Chieffi AL, Barata RCB. Ações judiciais: estratégia da indústria farmacêutica para introdução de novos medicamentos. Rev Saúde Pública 2010; 44:421-9.
7. Conselho Nacional de Justiça. Brasil tem mais de 240 mil processos na área de saúde. http://www. cnj.jus.br/noticias/cnj/14096-brasil-tem-mais-de-240-mil-processos-na-area-de-saude (acessado em 05/Ago/2011)

8. Herrera LHM. Judicialização das políticas públicas de assistência à saúde. Revista de Direito de Assistência à Saúde 2009; 16:73-91.

9. Wang DWL. Escassez de recursos, custos dos direitos e recursos, custos dos direitos e reserva do possível na jurisprudência do STF. Revista de Direito GV 2008; 4:539-68.

10. Messeder AM, Osório-de-Castro CGS, Luiza VL. Mandados judiciais como ferramenta para garantia do acesso a medicamentos no setor público: a experiência do Estado do Rio de Janeiro, Brasil. Cad Saúde Pública 2005; 21:525-34.

11. Chieffi AL, Barata RCB. Judicialização da política pública de assistência farmacêutica e equidade. Cad Saúde Pública 2009; 25:1839-49.

12. Macedo EL, Lopes LC, Barberato-Filho S. Análise técnica para a tomada de decisão do fornecimento de medicamentos pela via judicial. Rev Saúde Pública $2011 ; 45: 706-13$. 
13. Borges DCL, Ugá MAD. Conflitos e impasses da judicialização na obtenção de medicamentos: as decisões de 1ạ instância nas ações individuais contra o Estado do Rio de Janeiro, Brasil, em 2005. Cad Saúde Pública 2010; 26:59-69.

14. Campos Neto OH, Acurcio FA, Machado MAA, Ferré F, Barbosa FLV, Cherchiglia ML, et al. Médicos, advogados e indústria farmacêutica na judicialização da saúde em Minas Gerais, Brasil. Rev Saúde Pública 2012; 46:784-90.

15. Pereira JR, Santos RI, Nascimento Junior JM, Schenkel EP. Análise das demandas judiciais para o fornecimento de medicamentos pela Secretaria de Estado da Saúde de Santa Catarina nos anos de 2003 e 2004. Ciênc Saúde Coletiva 2010; 15: 3551-60.

16. Marques SB, Dallari SG. Garantia do direito social à assistência farmacêutica no Estado de São Paulo. Rev Saúde Pública 2007; 41:101-7.

17. Pepe VLE, Ventura M, Sant'ana JMB, Figueiredo TA, Souza VR, Simas L, et al. Caracterização de demandas judiciais de fornecimento de medicamentos "essenciais" no Estado do Rio de Janeiro, Brasil. Cad Saúde Pública 2010; 26:461-71.

18. Machado MAA. Judicialização do acesso a medicamentos no Estado de Minas Gerais, Brasil. Rev Saúde Pública 2011; 45:590-8.

19. Figueiredo TA, Pepe VLE, Osório-de-Castro CGS. Um enfoque sanitário sobre a demanda judicial de medicamentos. Physis (Rio J.) 2010; 20:101-18.

20. Bellato R, Araújo LFS, Nepomuceno AS, Mufato SLF, Souza GHL, Côrreas T. Mediação do direito à saúde pelo tribunal de justiça: análise da demanda. Texto \& Contexto Enferm 2012; 21:356-62.

21. Vieira FS, Zucchi P. Demandas judiciais e assistência terapêutica no Sistema Único de Saúde. Rev Assoc Méd Bras (1992) 2009; 55:672-82.

22. Fleury S. Judicialização pode salvar o SUS. Saúde Debate 2012; 93:159-62.

23. Ministério da Saúde. Manual técnico operacional do sistema de gerenciamento da Tabela de Procedimentos, Medicamentos e OPM do SUS. Brasília: Ministério da Saúde; 2011.

24. Travassos C, Martins M. Uma revisão sobre os conceitos de acesso e utilização de serviços de saúde. Cad Saúde Pública 2004; 20 Suppl 2:S190-8.

25. Ministério da Saúde. Índice de desempenho do Sistema Único de Saúde. http://portal.saude.gov. br/portal/saude/Gestor (acessado em 10/Out/ 2012).
26. Schramm JMA, Valente JG, Gadelha AMJ, Oliveira AF, Portela MC, Campos MR. Transição epidemiológica e o estudo de carga de doença no Brasil. Ciênc Saúde Coletiva 2004; 9:897-908.

27. Secretaria de Gestão Estratégica e Participativa, Ministério da Saúde. Regulamentação da Lei 8.080 para fortalecimento do Sistema Único da Saúde: decreto 7508, de 2011. Rev Saúde Pública 2011; 45:1206-7.

28. Brasil. Decreto no 7646, de 21 de dezembro de 2001. Dispõe sobre a Comissão Nacional de Incorporação de Tecnologias no Sistema Único de Saúde e sobre o processo administrativo para incorporação, exclusão e alteração de tecnologias em saúde pelo Sistema Único de Saúde - SUS, e dá outras providências. http://portal.saude.gov.br/ portal/arquivos/pdf/DECRETO_7646_CONITEC. pdf. (acessado em 10/Out/2012).

29. Ministério da Saúde. Portaria no $1101 /$ GM de 12 de junho de 2002. http://saude.gov.br/sas/PORTA RIAS/Port2002/Gm/GM-1101.htm (acessado em 10/Set/2012).

30. Conselho Federal de Medicina. Crise na assistência: falta de financiamento impacta no número de leitos e prejudica trabalho médico. http://portal. cfm.org.br (acessado em 13/Set/2012).

31. Campos GWS, Amaral MAA. A clínica ampliada e compartilhada, a gestão democrática e redes de atenção como referências teórico-operacionais para a reforma do hospital. Ciênc Saúde Coletiva 2007; 12:849-59.

32. Carvalho TC, Gianini RJ. Equidade no tempo de espera para determinadas cirurgias eletivas segundo o tipo de hospital em Sorocaba, SP. Rev Bras Epidemiol 2008; 11:473-83.

33. Instituto Brasileiro de Geografia e Estatística. PNAD: Pesquisa Nacional por Amostra de Domicílios [CD-ROM]. Rio de Janeiro: Instituto Brasileiro de Geografia e Estatística; 2003.

34. Campos CMS. Necessidade de saúde: uma análise da produção científica brasileira de 1990 a 2004. Interface Comun Saúde Educ 2007; 11:605-18.

35. Spedo SM, Silva Pinto NR, Tanaka OU. O difícil acesso a serviços de média complexidade do SUS: o caso da cidade de São Paulo, Brasil. Physis (Rio J.) 2010; 20:953-72.

Recebido em 11/Dez/2012

Versão final reapresentada em 25/Abr/2013

Aprovado em 24/Jul/2013 